\title{
Erratum to: Degree of naturalization of non-native plants on dumps
}

Oleg Kupriyanov ${ }^{1,2}$, Andrey Kupriyanov ${ }^{1, *}$ and Baurjan Turalin ${ }^{3}$

${ }^{1}$ The Federal Research Center of Coal and Coal Chemistry of Siberian Branch of the Russian Academy of Sciences

${ }^{2}$ Federal Research Center for Information and Computational Technologies of Siberian Branch of the Russian Academy of Sciences

${ }^{3}$ Aktobe Regional State University named after K. Zhubanov

Original article: BIO Web of Conferences 31, 00014 (2021),

https://doi.org/10.1051/bioconf/20213100014

The last names of the authors of the article (Oleg Kupiyanov ${ }^{1,2}$, Andrey Kupiyanov ${ }^{1, *}$ ) should be replaced with the following text: Oleg Kupriyanov ${ }^{1,2}$, Andrey Kupriyanov ${ }^{1, *}$ 